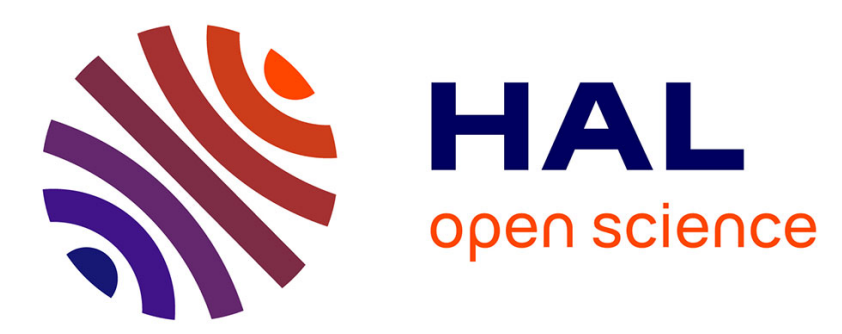

\title{
Measuring the spatial extent of individual localized photonic states
}

M. Spasenovic, D.M. Beggs, Philippe Lalanne, T.F. Krauss, L. Kuipers

\section{To cite this version:}

M. Spasenovic, D.M. Beggs, Philippe Lalanne, T.F. Krauss, L. Kuipers. Measuring the spatial extent of individual localized photonic states. Physical Review B: Condensed Matter and Materials Physics (1998-2015), 2012, 86 (15), pp.155153. 10.1103/PhysRevB.86.155153 . hal-00811649

\section{HAL Id: hal-00811649 \\ https://hal-iogs.archives-ouvertes.fr/hal-00811649}

Submitted on 19 Nov 2015

HAL is a multi-disciplinary open access archive for the deposit and dissemination of scientific research documents, whether they are published or not. The documents may come from teaching and research institutions in France or abroad, or from public or private research centers.
L'archive ouverte pluridisciplinaire HAL, est destinée au dépôt et à la diffusion de documents scientifiques de niveau recherche, publiés ou non, émanant des établissements d'enseignement et de recherche français ou étrangers, des laboratoires publics ou privés. 


\title{
Measuring the spatial extent of individual localized photonic states
}

\author{
Marko Spasenović, ${ }^{1}$ Daryl M. Beggs, ${ }^{1}$ Philippe Lalanne, ${ }^{2}$ Thomas F. Krauss, ${ }^{3}$ and L. Kuipers ${ }^{1}$ \\ ${ }^{1}$ Center for Nanophotonics, Foundation for Fundamental Research on Matter Institute for Atomic and Molecular Physics, \\ Science Park 104, 1098 XG Amsterdam, The Netherlands \\ ${ }^{2}$ Laboratoire Photonique, Numériques et Nanosciences, Institut d'Optique, Université Bordeaux 1, CNRS, 33405 Talence cedex, France \\ ${ }^{3}$ School of Physics and Astronomy, University of St. Andrews, North Haugh, Fife, KY16 9SS, United Kingdom \\ (Received 22 December 2011; revised manuscript received 27 September 2012; published 26 October 2012)
}

\begin{abstract}
We measure the spatial extent of individual photonic states that are localized by residual disorder in a slow-light photonic crystal waveguide. The size of the states is measured by locally perturbing them individually through an electromagnetic interaction with a near-field probe. We identify localized states that are not observed in transmission and show that they are shorter than the waveguide length. We also obtain near-field measurements of the participation ratio, from which the size of the states is also derived, in quantitative agreement with the size measured with the perturbation method.
\end{abstract}

PACS number(s): 42.70.Qs, 42.25.Dd, 78.67.Pt

Waves in disordered media can undergo multiple scattering, resulting in the formation of Anderson-localized states with an associated impeded wave transport. Anderson localization is a universal wave phenomenon, with manifestations in electron transport, ${ }^{1}$ sound, ${ }^{2}$ matter waves, ${ }^{3,4}$ and light. ${ }^{5,6}$ The spatial extent of localized states, or localization length, is of primary importance. For example, in systems of finite size, when this length is larger than the length of the sample, disorder has, on average, little effect on wave propagation. ${ }^{7}$ Conversely, when this length is smaller than the sample length, strongly confined states with typical lengths shorter than the sample size are likely to occur and wave transport may be severely disrupted. The localization length is an ensemble-averaged quantity, typically obtained by averaging over frequency or many realizations of disorder.

Here we show measurements of the spatial extent of individual localized photonic states for a single realization of disorder in a photonic crystal waveguide. To emphasize the difference between the ensemble-averaged localization length and the measured spatial extent of an individual localized state for a single optical frequency for a single realization of disorder, we will use the symbol $L_{\text {ind }}$ for the length that we measure. The spatial extent of localized photonic states has been measured before in a two-dimensional waveguide with embedded impurities. ${ }^{8}$ In that waveguide the spectral width of the localized resonances is on the order of $10 \mathrm{~nm}$. Close to the band edge of a photonic crystal waveguide, in the technologically important slow light region, localized resonances can have spectral widths on the order of $0.1 \mathrm{~nm}$, associated with small mode volumes. Measuring spectrally narrow resonances with a near-field microscope is a challenge because the tip of the near-field microscope influences the local dielectric constant in its vicinity. This dielectric constant has a paramount influence on the spectral position of a resonance. We show a method to measure the length of a localized state in a photonic crystal waveguide by using the spectral shift to our advantage. We measure $L_{\text {ind }}$ with two different methods, a local perturbation method and one based on the inverse participation ratio (IPR), and show that the results are in quantitative agreement. We identify states for which $L_{\text {ind }}$ is smaller than the length of the waveguide and show that these states are not observable in traditional transmission measurements, although such states with their small volumes are arguably the most useful for applications in quantum computing and sensing.

The idea that disorder in photonic crystals can be used as a model system for the study of localization is as old as the research field of photonic crystals itself, ${ }^{9}$ and photonic crystal waveguides have been shown to be a good example of truly disordered systems in which Anderson localization occurs. $^{10,11}$ For one-dimensional (1D) periodic systems, localization always occurs for any nonzero disorder, a fact well established for electrons in 1D potentials. ${ }^{1}$ As a model system we investigate a $1 \mathrm{D}$ photonic crystal waveguide (PCW) for a range of frequencies (wavelengths) near the band edge, as depicted in Fig. 1(a). The calculated dispersion of our PCW is shown in Fig. 1(b). At wavelengths close to $1567.5 \mathrm{~nm}$, the slope of the dispersion becomes shallow, indicating a decreased group velocity and an increase in the photonic density of states.

Although our PCW is fabricated with state-of-the-art techniques to nominally ideal designs, some residual disorder always remains, resulting in photon scattering. The amount of backscattering into the waveguide scales with the density of states, proportional to the group index squared, ${ }^{12}$ and so increases dramatically near the band edge. ${ }^{13}$ Backscattering dominates in the slow-light/multiple scattering regime, as it scales with $n_{g}^{2}$, whereas out-of-plane scattering only increases with $n_{g}{ }^{12}$ Since the localization length decreases with increasing backscattering, ${ }^{7}$ near the band edge the localization length may be expected to decrease. For typical device lengths $(\approx 100 \mu \mathrm{m})$, multiple scattering starts to dominate the transport at group indices $n_{g} \approx 65,{ }^{11,13}$ depending on the fabrication quality.

Localized states of light arising from disorder possess a resonance behavior similar to that of engineered photonic microcavities. ${ }^{14}$ In photonic crystals such microcavities consist of deliberately engineered defects that confine light to small volumes with high quality factors. ${ }^{15}$ In Anderson localization such cavities form through the wave interference resulting from multiple scattering by random configurations of disorder in the waveguide. As such, the individual cavities occur at unpredictable positions, with unpredictable quality factors and resonance frequencies. To investigate these 

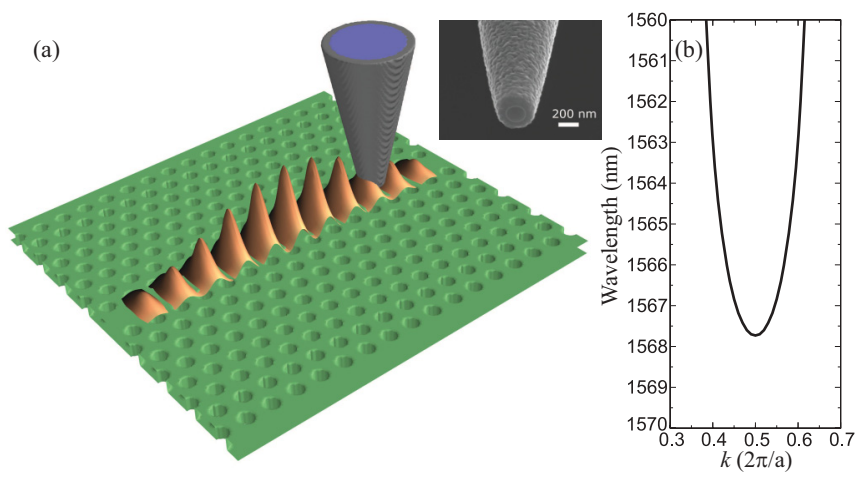

FIG. 1. (Color online) Schematic representation of the sample and near-field probe. (a) An aluminum-coated near-field probe (inset) is scanned over the surface and collects a fraction of the light. The magnetic field of light interacts with the aluminum coating to shift the resonance of localized states. (b) Calculated dispersion curve for the nominal design.

Anderson localized states we use a near-field probe consisting of a tapered glass fiber of diameter $\sim 200 \mathrm{~nm}$, coated with aluminum of thickness $\sim 150 \mathrm{~nm}$ [Fig. 1(a), inset]. Usually, in near-field microscopy, such a probe is introduced into the evanescent field of an optical mode and the electric field of light in photonic eigenstates is detected with high resolution without significantly perturbing the states. ${ }^{16}$ Above a photonic crystal cavity with a reasonably high quality factor, however, the probe may shift the frequency of the states due to the light-matter interaction between it and the light in the cavity. Previous studies have introduced such probes into the near field of engineered photonic crystal microcavities, deducing properties of the cavity and/or probe from the well defined shifts of the cavity resonance. ${ }^{17-19}$ The electric part of the interaction typically leads to an increase in the effective volume of the cavity, resulting in a redshift of the resonance, the magnitude of which is inversely proportional to the volume of the photonic state. ${ }^{17}$ In the magnetic part of the interaction, a nanoscopic manifestation of Lenz's law causes an induced magnetic field that opposes the magnetic field of light in the cavity, resulting in a blueshift of the resonance frequency of the cavity, again with a magnitude inversely proportional to the volume of the state. ${ }^{18,19}$ This magnetic interaction can be larger than the electric one ${ }^{18}$ resulting in an overall blueshift of the resonance frequencies. By inserting the probe into the near field of the localized states and measuring the resultant blueshift we will determine the state volume and thus measure $L_{\text {ind }}$ directly on each instance of localization.

First we measure a transmission spectrum of the unperturbed waveguide, with the probe far from the sample [Fig. 2(a)]. Three spectral regions can be discerned. For a freespace wavelength $\lambda \lesssim 1561.6 \mathrm{~nm}$, the transmission is high, as light is transported through the modes of the waveguide. For $1561.6 \mathrm{~nm} \lesssim \lambda \lesssim 1563.2 \mathrm{~nm}$ the transmission drops, but many large and wide peaks are apparent. Finally, for $\lambda \gtrsim$ $1563.2 \mathrm{~nm}$, the transmission is very low, with a few narrow (typical width of $0.01 \mathrm{~nm}$ ) and sparse peaks appearing, typically a manifestation of Anderson localization. ${ }^{20,21}$

Next we scan our near-field probe in the near field of the sample and measure the electric field above the waveguide.
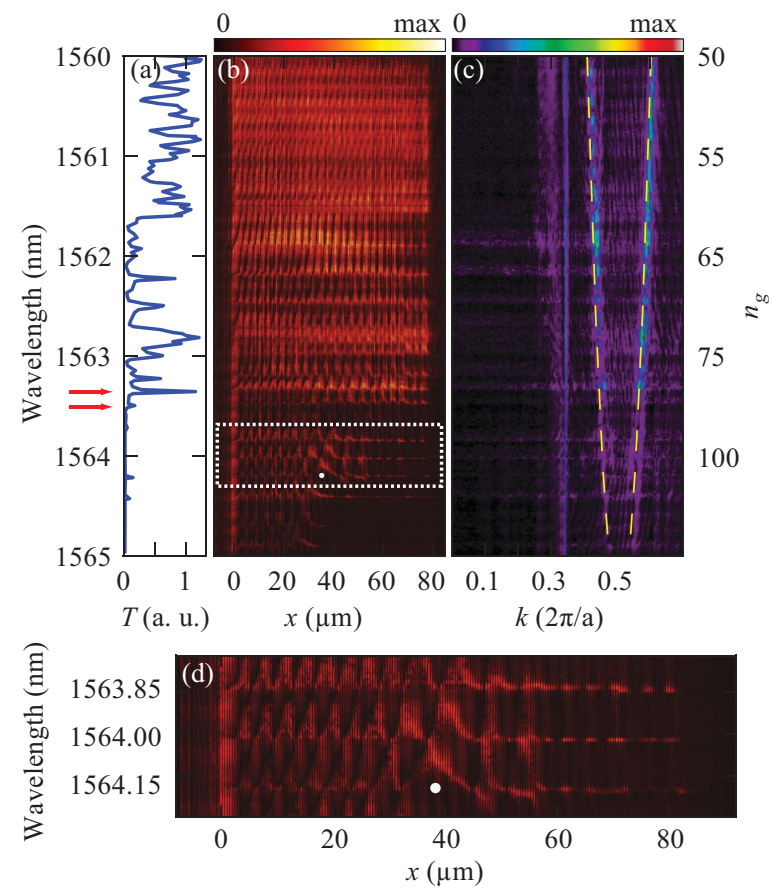

FIG. 2. (Color online) Waveguide transmission, near-field amplitude, and dispersion. (a) Transmission spectrum $T$ of the PCW. (b) Near-field amplitude collected through the probe as a function of wavelength and probe position along the center of the waveguide. Light enters the PCW from the left, at position $x=0$. (c) Band structure of the waveguide. Waveguide modes appear as lines in the spectrum. The almost vertical line around $k=0.33$ is the light line in the silicon slab. (d) Closeup of the near-field region indicated with a dotted white box in (b).

After the electric field is measured over the length of the waveguide, we change the wavelength with a step of $0.01 \mathrm{~nm}$. The amplitude of the electric field, as a function of laser wavelength and probe position, is depicted in Fig. 2(b). We observe that the magnitude of the measured electric field at the exit of the PCW (position $x=82 \mu \mathrm{m}$ ) correlates with the measured far-field transmission for all wavelengths: For $\lambda \lesssim 1561.6 \mathrm{~nm}$ (top of the image), we operate far above the band edge, light propagates in the Bloch modes of the periodic structure, and the electric-field amplitude at the exit is similar to that at the entrance. For $1561.6 \mathrm{~nm} \lesssim \lambda \lesssim 1563.2 \mathrm{~nm}$, the magnitude of the measured electric field at the exit decreases on average. However, we also occasionally observe sharp increases in the amplitude in the waveguide, corresponding to the localized states that span the length of the sample (see, for example, close to $\lambda \approx 1562.0$ or $1563.35 \mathrm{~nm}$ ). At longer wavelengths $(\lambda \gtrsim 1563.2 \mathrm{~nm})$, instances of populated states become rare. Nevertheless, we observe light located and localized inside the waveguide at wavelengths for which transmission is negligible. At these wavelengths, the light has more intensity close to the entrance of the waveguide than at the exit, indicating localized states that are shorter than the waveguide and are populated by the light incident from the input waveguide on the left. Since the states are populated from the left and are shorter than the waveguide, the intensity at transmission on the right is small. States that are localized on the right side of the waveguide could 
also be excited, although with less efficiency. However, the measured amplitude of such states would be small because they would have good coupling to the output waveguide. At long wavelengths, the bandwidth of localized states decreases due to an increasing $Q$ factor. ${ }^{11}$ As we approach the band edge, we expect the bandwidth to decrease below the spectral resolution of our laser and thus we expect that there exist states that we fail to excite or that some states are excited away from the center of the peak. In either case, there is no reason to believe that the bandwidth has an influence on the analysis and interpretation that follow. It is noteworthy that the periodic amplitude modulation owing to beating between forward- and backward-propagating Bloch modes persists throughout the localization regime, an indication that at all frequencies the light retains much of the Bloch nature of the nominal, perfect structure despite being multiply scattered.

Figure 2(c) depicts the dispersion of the waveguide mode, obtained via a Fourier transform of the real-space data. ${ }^{22}$ The measured dispersion curve of the photonic crystal waveguide follows the behavior expected from calculation [Fig. 1(b)]. It is noteworthy that the measured band structure follows that of the nominal perfect structure throughout the localization regime. In the band structure, horizontal lines spanning reciprocal space indicate the presence of localized states; the broad distribution of wave vectors results from the high spatial confinement. Away from the band edge (top of the image), the dispersion curve can be approximated by a parabola (dashed yellow line). The group index, shown on the right-hand axis, is obtained by taking the first-order derivative of the fit. Theory and measurements involving statistical averaging ${ }^{11}$ have shown that the average length of localization instances, for the batch of samples from which this waveguide was taken, becomes of the order of the waveguide length at $n_{g} \sim 65$. Although the appearance of localization instances is a random process, this group index corresponds very well with our first observation of a localized state, at $\lambda=1562 \mathrm{~nm}$.

As the near-field probe collects the electric field with high resolution, we observe its interaction with the electric and magnetic fields as shifts in the spectral position of the localized resonances. This shifting is most evident for long wavelengths $(\lambda>1563.5 \mathrm{~nm})$, i.e., at the bottom of Fig. 2(b). We observe that for certain spatial positions of the probe, the maximum of the collected electric field is shifted from its original spectral position towards shorter wavelengths. One such position is indicated by a white dot in the image. A closeup of the region with the strongest shifts, indicated by a dotted white box in Fig. 2(b), is given in Fig. 2(d). The spatial periodicity of the shift highlights the remnants of the Bloch nature of the localized states.

For engineered photonic crystal microcavities, using a rigorous solution of Maxwell's equations, the spectral shift was shown to be given by ${ }^{17,18}$

$$
\begin{aligned}
\frac{\Delta \lambda\left(\mathbf{r}_{\mathrm{pr}}\right)}{\lambda} & \approx \alpha_{\mathrm{eff}}^{e} \frac{\left|E_{0}\right|^{2}}{U_{0}}+\alpha_{\mathrm{eff}}^{m} \frac{\left|B_{0}\right|^{2}}{U_{0}} \\
& =\frac{\alpha_{\mathrm{eff}}^{e}}{2 \epsilon_{0} V_{\mathrm{cav}}} \beta_{E}+\frac{\alpha_{\mathrm{eff}}^{m}}{2 \mu_{0} V_{\mathrm{cav}}} \beta_{H},
\end{aligned}
$$

where $U_{0}$ is the energy of the unperturbed cavity field; $E_{0}$ and $B_{0}$ are, respectively, the total electric and magnetic fields of the unperturbed cavity; $\alpha_{\mathrm{eff}}^{e}$ is the effective polarizability of the probe; $\alpha_{\text {eff }}^{m}$ is the magnetic polarizability of the probe; $V_{\text {cav }}$ is the cavity volume [(area) $\times$ (length)]; $\epsilon_{0}$ is the permittivity of free space; $\mu_{0}$ is the magnetic constant; $\beta_{E}$ is the ratio of the intensity the electric field at the probe position $\left|\mathbf{E}\left(\mathbf{r}_{\mathrm{pr}}\right)\right|^{2}$ to its maximum in the sample $\max |\mathbf{E}|^{2}$; and $\beta_{H}$ is the ratio of the magnetic-field component pointing into the probe $\left|H_{z}\left(\mathbf{r}_{\mathrm{pr}}\right)\right|^{2}$ to its maximum in the sample $\max \left|H_{z}\right|^{2}$.

The electric and magnetic polarizabilities of our probes were measured by Burresi et al. ${ }^{18}$ to have values of $\alpha_{\text {eff }}^{e}=3 \times 10^{-21} \mathrm{~m}^{3} \epsilon_{0} \quad$ and $\quad \alpha_{\text {eff }}^{m}=-12 \times 10^{-21} \mathrm{~m}^{3} / \mu_{0}$, respectively. The probes used here are similar to those of Burresi et al. (i.e., same aperture size, coating thickness, etc.). To estimate the other parameters in Eq. (1) we calculated the eigenmodes of the unperturbed photonic crystal waveguide by using the MIT Photonic-Bands Package. ${ }^{23}$ The eigenmodes at the wavelengths of our study have an effective cross-sectional area $A=1.12 a^{2}$ (with $a$ the period of the photonic crystal lattice) and the ratios $\beta_{E}=\beta_{H}=0.22$ at the height of the probe. We calculate with modes of the unperturbed waveguide because measurements of dispersion indicate that even in the localization regime, the light populates Bloch modes of the waveguide. It has been shown before, through calculations that use only modes of the unperturbed waveguide, that the area that light occupies in a PCW spreads as expected. ${ }^{24,25}$ Also, eigenmodes of the unperturbed system were used in studies of losses due to roughness in PCWs. ${ }^{26}$ We are now in a position to determine the length of each individual localized state $L_{\text {ind }}$ as

$$
L_{\text {ind }}=0.22 \frac{\lambda}{\Delta \lambda} \frac{\alpha_{\mathrm{eff}}^{e}+\alpha_{\mathrm{eff}}^{m}}{2 A},
$$

where we have used $V_{\text {cav }}=L_{\text {ind }} A$ and, because of the persistent Bloch nature of light, we take $A$ to be the same as that of the unperturbed Bloch mode, see Supplemental Material. ${ }^{27}$ We present these measured localization lengths as a function of wavelength and group index by the blue points in Fig. 3 . These measurements of the individual localized state length in a disordered microphotonic system are shown directly. As expected, the localization length generally becomes smaller at higher $n_{g}$, as the backscattering of waves increases. ${ }^{12}$ With our method we can precisely determine that for this particular

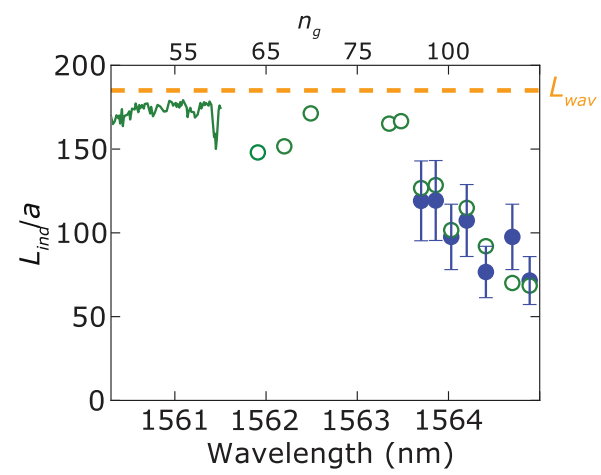

FIG. 3. (Color online) Dispersion of the localization length. The blue dots are points measured with the near-field interaction method. The green data points are measured with the IPR method. The horizontal dashed line indicates $L_{\text {wav }}$, the length of the waveguide. 
waveguide, multiple scattering and Anderson localization dominate transport when $L_{\text {ind }}<L_{\text {wav }}$, at $n_{g}>80$. At higher nominal values of $n_{g}$, we still observe localized states in the near field of the waveguide, but they are significantly shorter than the waveguide and do not contribute to transmission.

Alternatively, we can also determine the degree of localization by measuring the IPR, also in the near field, as the IPR is proportional to localization length. ${ }^{28}$ The IPR is a quantity related to $L_{\text {ind }}$, often used as a measure of disorder. For example, the ensemble-averaged IPR has been used to measure quantum eigenfunctions in ensembles of metallic grains, ${ }^{29}$ disorder in two-dimensional photonic lattices, ${ }^{30}$ and to diagnose malignancy in biological cells. ${ }^{31}$ In one dimension and normalized to the length of the waveguide, the IPR $\mathcal{I}$ takes the form

$$
\mathcal{I} \propto \frac{1}{L_{\text {ind }}}=\frac{\int|\mathbf{E}(\mathbf{r})|^{4} d \mathbf{r}}{\left(\int|\mathbf{E}(\mathbf{r})|^{2} d \mathbf{r}\right)^{2}} .
$$

As we have measured the electric field as a function of position, we determine $L_{\text {ind }}$ using Eq. (3). We plot the $L_{\text {ind }}$ obtained with this IPR method in green in Fig. 3. We find excellent quantitative agreement between the two methods. Taking Figs. 2 and 3 as a whole, we now conclude that three transport regimes can be identified. In the regime at the lowest frequencies, where an $L_{\text {ind }}$ as small as approximately $L_{\text {wav }} / 3$ is found, populated localized states do exist with lengths significantly shorter than the length of the waveguide. It is exactly these states that are useful for applications requiring strongly localized fields, such as quantum optics. ${ }^{10}$ In the next regime, for wavelengths decreased away from the band edge, the localization length increases and becomes on the order of the waveguide length at $\lambda \approx 1563.5 \mathrm{~nm}\left(n_{g} \approx 80\right)$. Here sharp dips in transmission occur, indicating nondeterministic instances of $L_{\text {ind }}<L_{\text {wav }}$, and photon transport becomes unpredictable, with almost equal probability of high or low transmission at any given wavelength. In the third regine, $\lambda<156 \mathrm{~nm}\left(n_{g}<55\right), L_{\text {ind }} \approx L_{\text {wav }}$ for all wavelengths, indicating extended states. In all regimes of transport, our method of measuring the $L_{\text {ind }}$ from the probe-field interaction gives the same result as the IPR method, which is commonly used to measure the statistically averaged degree of disorder in various systems. ${ }^{30,31}$ Note that in disordered systems, spectrally overlapping resonances known as necklace states sometimes occur. ${ }^{20,32}$ Such an occurrence in our system would result in a measurement of the combined length of the overlapping states rather than measurements of individual state length. Although we do not exclude the possibility that we observe necklace states, it is highly unlikely given that these states are extremely rare in long samples ${ }^{20}$ and that we use only one sample.

Our work shows that a key parameter in localization studies can be measured directly for a single realization of disorder. The $L_{\text {ind }}$ of a single instance cannot be measured by observing transmission and states for which $L_{\text {ind }}<L_{\text {wav }}$ are often not even observable in transmission. Our measurement approach can be extended to investigate and understand the behavior of any disordered system where electromagnetic waves are present at the surface. The technique can easily be extended to finite-size 2D systems, which are far from trivial and in which the interplay between localization and out-of-plane scattering still has to be fully understood. Notably, we do not need to perform the measurement over the entire length or volume of the state in order to determine $L_{\text {ind }}$. Our method could thus be used to determine the volume of localized states just below the surface of a 3D disordered (photonic crystal) structure. From an application point of view, a recent study shows that localization in photonic crystal waveguides can initiate lasing from quantum dots. ${ }^{33}$ The gain of random lasers has been theoretically predicted to depend exponentially on localization length, ${ }^{34}$ but shown numerically to have a power-law dependence on localization length. ${ }^{35}$ Our technique provides an ideal method to resolve such dual predictions by allowing studies of gain and state length for each individual localized state. We further expect our local perturbation method to also work for other wave phenomena such as acoustics. The fact that we exploit the magnetically induced light-matter interaction also reveals the fascinating opportunity to study disorder in systems with a strong magnetic field at optical frequencies, such as metamaterials. ${ }^{36,37}$

Note added. We have recently become aware of similar work by Huisman et al. ${ }^{38}$

We thank Ad Lagendijk, Femius Koenderink, and Sanli Faez for useful discussions. This work is part of the research program of the Stichting voor Fundamenteel Onderzoek der Materie, which is financially supported by the Nederlandse Organisatie voor Wetenschappelijk Onderzoek. We thank the EC Marie Curie Scheme (Contract No. MEST-CT-2005021000). The authors acknowledge support from the Smart Mix Programme of the Netherlands Ministry of Economic Affairs and the Netherlands Ministry of Education, Culture and Science.
${ }^{1}$ P. W. Anderson, Phys. Rev. 109, 1492 (1958).

${ }^{2}$ H. Hu, A. Strybulevych, J. H. Page, S. E. Skipetrov, and B. A. van Tiggelen, Nat. Phys. 4, 945 (2008).

${ }^{3}$ J. Billy, V. Josse, Z. Zuo, A. Bernard, B. Hambrecht, P. Lugan, D. Clement, L. Sanchez-Palencia, P. Bouyer, and A. Aspect, Nature (London) 453, 891 (2008).

${ }^{4}$ G. Roati, C. D'Errico, L. Fallani, M. Fattori, C. Fort, M. Zaccanti, G. Modugno, M. Modugno, and M. Inguscio, Nature (London) 453, 895 (2008).
${ }^{5}$ D. S. Wiersma, P. Bartolini, A. Lagendijk, and R. Righini, Nature (London) 390, 671 (1997).

${ }^{6}$ C. M. Aegerter, M. Störzer, and G. Maret, Europhys. Lett. 75, 562 (2006).

${ }^{7}$ S. Mazoyer, J. P. Hugonin, and P. Lalanne, Phys. Rev. Lett. 103, 063903 (2009).

${ }^{8}$ F. Riboli, P. Barthelemy, S. Vignolini, F. Intonti, A. De Rossi, S. Combrie, and D. S. Wiersma, Opt. Lett. 36, 127 (2011).

${ }^{9}$ S. John, Phys. Rev. Lett. 58, 2486 (1987). 
${ }^{10}$ L. Sapienza, H. Thyrrestrup, S. Stobbe, P. D. García, S. Smolka, and P. Lodahl, Science 327, 1352 (2010).

${ }^{11}$ S. Mazoyer, P. Lalanne, J. Rodier, J. Hugonin, M. Spasenović, L. Kuipers, D. Beggs, and T. F. Krauss, Opt. Express 18, 14654 (2010).

${ }^{12}$ E. Kuramochi, M. Notomi, S. Hughes, A. Shinya, T. Watanabe, and L. Ramunno, Phys. Rev. B 72, 161318 (2005).

${ }^{13}$ R. J. P. Engelen, D. Mori, T. Baba, and L. Kuipers, Phys. Rev. Lett. 101, 103901 (2008).

${ }^{14}$ J. B. Pendry, Physics 1, 20 (2008).

${ }^{15}$ Y. Akahane, T. Asano, B.-S. Song, and S. Noda, Nature (London) 425, 944 (2003).

${ }^{16}$ E. Betzig, M. Isaacson, and A. Lewis, Appl. Phys. Lett. 51, 2088 (1987).

${ }^{17}$ A. F. Koenderink, M. Kafesaki, B. C. Buchler, and V. Sandoghdar, Phys. Rev. Lett. 95, 153904 (2005).

${ }^{18}$ M. Burresi, T. Kampfrath, D. van Oosten, J. C. Prangsma, B. S. Song, S. Noda, and L. Kuipers, Phys. Rev. Lett. 105, 123901 (2010).

${ }^{19}$ S. Vignolini, F. Intonti, F. Riboli, L. Balet, L. H. Li, M. Francardi, A. Gerardino, A. Fiore, D. S. Wiersma, and M. Gurioli, Phys. Rev. Lett. 105, 123902 (2010).

${ }^{20}$ J. Bertolotti, S. Gottardo, D. S. Wiersma, M. Ghulinyan, and L. Pavesi, Phys. Rev. Lett. 94, 113903 (2005).

${ }^{21}$ P. Sebbah, B. Hu, J. M. Klosner, and A. Z. Genack, Phys. Rev. Lett. 96, 183902 (2006).

${ }^{22}$ R. J. P. Engelen, Y. Sugimoto, H. Gersen, N. Ikeda, K. Asakawa, and L. Kuipers, Nat. Phys. 3, 401 (2007).

${ }^{23}$ S. Johnson and J. Joannopoulos, Opt. Express 8, 173 (2001).

${ }^{24}$ S. Ha, M. Spasenović, A. A. Sukhorukov, T. P. White, C. M. de Sterke, L. Kuipers, T. F. Krauss, and Y. S. Kivshar, J. Opt. Soc. Am. B 28, 955 (2011).
${ }^{25}$ M. Spasenović, T. P. White, S. Ha, A. A. Sukhorukov, T. Kampfrath, Y. S. Kivshar, C. M. de Sterke, T. F. Krauss, and L. Kuipers, Opt. Lett. 36, 1170 (2011).

${ }^{26}$ S. G. Johnson, M. L. Povinelli, M. Soljačić, A. Karalis, S. Jacobs, and J. D. Joannopoulos, Appl. Phys. B 81, 283 (2005).

${ }^{27}$ See Supplemental Material at http://link.aps.org/supplemental/ 10.1103/PhysRevB.86.155153 for two different methods of measuring the spectral shift of the resonances, one method using changes in transmission, and the other method using statistical averaging of resonance shifts along the length of the waveguide.

${ }^{28}$ M. Schreiber, Phys. Rev. B 31, 6146 (1985).

${ }^{29}$ V. N. Prigodin and B. L. Altshuler, Phys. Rev. Lett. 80, 1944 (1998).

${ }^{30}$ T. Schwartz, G. Bartal, S. Fishman, and M. Segev, Nature (London) 446, 52 (2007).

${ }^{31}$ H. Subramanian, P. Pradhan, Y. Liu, I. R. Capoglu, X. Li, J. D. Rogers, A. Heifetz, D. Kunte, H. K. Roy, A. Taflove, and Vadim Backman, Proc. Natl. Acad. Sci. USA 105, 20118 (2008).

${ }^{32}$ A. V. Tartakovskii, M. V. Fistul, M. E. Raikh, and I. M. Ruzin, Sov. Phys. Semicond. 21, 370 (1987).

${ }^{33}$ J. K. Yang, H. Noh, M. J. Rooks, G. S. Solomon, F. Vollmer, and H. Cao, Appl. Phys. Lett. 98, 241107 (2011).

${ }^{34}$ A. L. Burin, M. A. Ratner, H. Cao, and S. H. Chang, Phys. Rev. Lett. 88, 093904 (2002).

${ }^{35}$ Q. M. Li, K. M. Ho, and C. M. Soukoulis, Physica B 296, 78 (2001).

${ }^{36} \mathrm{~S}$. Linden, C. Enkrich, M. Wegener, J. Zhou, T. Koschny, and C. M. Soukoulis, Science 306, 1351 (2004).

${ }^{37}$ M. Burresi, D. van Oosten, T. Kampfrath, H. Schoenmaker, R. Heideman, A. Leinse, and L. Kuipers, Science 326, 550 (2009).

${ }^{38}$ S. R. Huisman, G. Ctistis, S. Stobbe, A. P. Mosk, J. L. Herek, A. Lagendijk, P. Lodahl, W. L. Vos, and P. W. H. Pinkse, following paper, Phys. Rev. B 86, 155154 (2012). 